\title{
On semiconcise words
}

\section{Costantino Delizia, Pavel Shumyatsky and Antonio Tortora}

\author{
Communicated by Evgenii I. Khukhro
}

\begin{abstract}
Let $w$ be a group-word. For a group $G$, let $G_{w}$ denote the set of all $w$-values in $G$ and $w(G)$ the verbal subgroup of $G$ corresponding to $w$. The word $w$ is semiconcise if the subgroup $[w(G), G]$ is finite whenever $G_{w}$ is finite. The group $G$ is an $\operatorname{FC}(w)$-group if the set of conjugates $x^{G}$ is finite for all $x \in G$. We prove that if $w$ is a semiconcise word and $G$ is an $\mathrm{FC}(w)$-group, then the subgroup $[w(G), G]$ is FC-embedded in $G$, that is, the intersection $C_{G}(x) \cap[w(G), G]$ has finite index in $[w(G), G]$ for all $x \in G$. A similar result holds for $\operatorname{BFC}(w)$-groups, that are groups in which the sets $x^{G_{w}}$ are boundedly finite. We also show that this is no longer true if $w$ is not semiconcise.
\end{abstract}

\section{Introduction}

Let $w=w\left(x_{1}, \ldots, x_{n}\right)$ be a group-word in the variables $x_{1}, \ldots, x_{n}$. For any group $G$ and arbitrary $g_{1}, \ldots, g_{n} \in G$, the elements of the form $w\left(g_{1}, \ldots, g_{n}\right)$ are called the $w$-values in $G$. The set of all $w$-values in $G$ is denoted by $G_{w}$. Clearly, any conjugate of a $w$-value is again a $w$-value, and so $G_{w}$ is a normal set. The verbal subgroup of $G$ corresponding to $w$ is the subgroup $w(G)$ generated by all $w$-values in $G$.

A word $w$ is called concise if the verbal subgroup $w(G)$ is finite in each group $G$ such that $G_{w}$ is finite. In the sixties, P. Hall conjectured that every word is concise, but his conjecture was refuted in 1989 by S. Ivanov ([8], see also [9]). However, many words of common use are known to be concise (see, e.g., [3, 6, 10,11]).

Let $w$ be any group-word, and suppose that $G_{w}$ is finite for a group $G$. It is well known that the derived group $w(G)^{\prime}$ is always finite and its order is bounded by a function depending only on the order of $G_{w}$. In [6], it has been proved that the same is true for the subgroup $[w(G), G]$, when $w$ is the $n$-Engel word

$$
[x, n y]=[x, \underbrace{y, \ldots, y}_{n}] .
$$

This work was partially supported by the "National Group for Algebraic and Geometric Structures, and their Applications" (GNSAGA - INdAM). The last author is also supported by a grant of the University of Campania "Luigi Vanvitelli", in the framework of Programma V:ALERE 2019. 
Motivated by this, we say that a word $w$ is semiconcise if the finiteness of $G_{w}$ for a group $G$ always implies the finiteness of the subgroup $[w(G), G]$.

Of course, Engel words and concise words are semiconcise. Moreover, if $w$ is a semiconcise word and $z$ is any variable not appearing in $w$, then the word $[w, z]$ is semiconcise (see Proposition 4.2). Thus the word $[x, n y, z]$ is semiconcise. So far, we do not know whether there exists a semiconcise word which is not concise. A suitable modification of Ivanov's example, given in [1], shows that there exists a word which is not semiconcise (see Section 4).

Further information on the subgroup $[w(G), G]$ when $w$ is a semiconcise word can be obtained by using a verbal generalization of FC-groups, namely groups with finite conjugacy classes. For subsets $X$ and $Y$ of a group $G$, we write $X^{Y}$ to denote the set of conjugates $\left\{x^{y} \mid x \in X, y \in Y\right\}$. A subgroup $H$ of a group $G$ is said to be FC-embedded in $G$ if $x^{H}$ is finite for all $x \in G$. The subgroup $H$ is BFC-embedded in $G$ if $x^{H}$ is finite for all $x \in G$ and the number of elements in $x^{H}$ is bounded by a constant that does not depend on the choice of $x$.

For any group-word $w$, a group $G$ is said to be an $\operatorname{FC}(w)$-group if $x^{G_{w}}$ is finite for all $x \in G$, and a $\operatorname{BFC}(w)$-group if $x^{G} w$ is finite for all $x \in G$ and the number of elements in $x^{G_{w}}$ is bounded by a constant that does not depend on the choice of $x$. Obvious examples of $\mathrm{FC}(w)$-groups (respectively, $\mathrm{BFC}(w)$-groups) are provided by groups $G$ in which the verbal subgroup $w(G)$ is FC-embedded (respectively, BFC-embedded) in G. On the other side, it has been proved in [7] (respectively, in [1]) that if $w$ is a concise word and $G$ is an $\mathrm{FC}(w)$-group (respectively, a $\operatorname{BFC}(w)$-group), then the verbal subgroup $w(G)$ is FC-embedded (respectively, BFC-embedded) in $G$.

In [2], for an arbitrary group-word $w$, we proved that if $G$ is an $\mathrm{FC}(w)$-group (respectively, a BFC-group), then $w(G)^{\prime}$ is FC-embedded (respectively, BFC-embedded) in $G$. In this paper, we restrict our attention to semiconcise words. More precisely, we prove the following theorems.

Theorem A. Let $w$ be a semiconcise word, and let $G$ be an $\mathrm{FC}(w)$-group. Then $[w(G), G]$ is FC-embedded in $G$.

Theorem B. Let $w$ be a semiconcise word, and let $G$ be a $\operatorname{BFC}(w)$-group. Then $[w(G), G]$ is BFC-embedded in $G$.

We also show that, for a certain word $w$ which is not semiconcise, there is an example of a $\operatorname{BFC}(w)$-group $G$ such that $[w(G), G]$ is not FC-embedded in $G$ (see Proposition 4.4). 


\section{Proof of Theorem A}

For a subset $S$ of a group $G$, write $S^{*}=S \cup S^{-1}$. Obviously, if $S$ is a normal set, then $S^{*}$ is normal too. Moreover, if $S$ is a finite set, we often refer to the "order of $S$ ", denoted by $|S|$, to mean "the number of elements in $S$ ".

Lemma 2.1. Let $w$ be a group-word. If $G$ is an $\mathrm{FC}(w)$-group, then the conjugacy class $x^{G_{w}^{*}}$ is finite for all $x \in G$.

Proof. By the first statement in [2, Proposition 2.9], a group $G$ is an $\mathrm{FC}(w)$-group if and only if it is an $\mathrm{FC}\left(w^{-1}\right)$-group. For all $g \in G$, we have $g \in G_{w}$ if and only if $g^{-1} \in G_{w^{-1}}$. Thus $G_{w^{-1}}=\left(G_{w}\right)^{-1}$, and the result follows.

Lemma 2.2. Let $w$ be a group-word, and let $G$ be an $\mathrm{FC}(w)$-group. Choose $x \in G$, and denote by $A$ a finite subset of $G_{w}^{*}$ such that $x^{G_{w}^{*}}=x^{A}$. Then, for any $j \geq 1$ and $y_{1}, \ldots, y_{j} \in G_{w}^{*}$, there exist $a_{1}, \ldots, a_{j} \in A$ such that $x^{y_{1} \ldots y_{j}}=x^{a_{1} \ldots a_{j}}$.

Proof. We argue by induction on $j$. The case $j=1$ is clear. Let $j>1$, and assume that $x^{y_{1} \ldots y_{j-1}}=x^{a_{1} \ldots a_{j-1}}$ with $a_{1}, \ldots, a_{j-1} \in A$. Then

$$
x^{y_{1} \ldots y_{j}}=x^{a_{1} \ldots a_{j-1} y_{j}}=x^{y_{j}^{b} a_{1} \ldots a_{j-1}},
$$

where $b=\left(a_{1} \ldots a_{j-1}\right)^{-1}$. Since $y_{j}^{b} \in G_{w}^{*}$, we have $x^{y_{j}{ }^{b}}=x^{a_{j}}$ for some $a_{j} \in A$, and so $x^{y_{1} \ldots y_{j}}=x^{a_{j} a_{1} \ldots a_{j-1}}$. After renumbering the elements $a_{i} \in A$, we obtain the required result.

Lemma 2.3. Let $w=w\left(x_{1}, \ldots, x_{n}\right)$ be a group-word, and set

$$
v=\left[w\left(x_{1}, \ldots, x_{n}\right), x_{n+1}\right]
$$

If $G$ is an $\mathrm{FC}(w)$-group, then it is an $\mathrm{FC}(v)$-group.

Proof. Let $y \in G_{v}$. Then there exist $g_{1}, \ldots, g_{n}, g_{n+1} \in G$ such that $y=z t$, with $z=w\left(g_{1}, \ldots, g_{n}\right)^{-1} \in\left(G_{w}\right)^{-1}=G_{w^{-1}}$ and $t=w\left(g_{1}, \ldots, g_{n}\right)^{g_{n+1}} \in G_{w}$. By Lemma 2.2, for any $x \in G$, the conjugate $x^{y}$ can only take finitely many values. This proves the result.

Lemma 2.4. Let $w=w\left(x_{1}, \ldots, x_{n}\right)$ be a semiconcise $w o r d$, and set

$$
v=\left[w\left(x_{1}, \ldots, x_{n}\right), x_{n+1}\right]
$$

Let $G$ be an $\mathrm{FC}(w)$-group and $B$ a finite subset of $G_{v}^{*}$. Then, for any $x \in G$, there exists a positive integer $e$ such that $b^{e} \in Z(\langle x, B\rangle)$ for all $b \in B$. 
Proof. Write $B=\left\{b_{1}, \ldots, b_{r}\right\}$, and let $x$ be an arbitrary element of $G$. For any $b_{i} \in B$, there exist elements $g_{i_{1}}, \ldots, g_{i_{n}}, g_{i_{n+1}} \in G$ such that either

$$
b_{i}=\left[w\left(g_{i_{1}}, \ldots, g_{i_{n}}\right), g_{i_{n+1}}\right] \quad \text { or } \quad b_{i}=\left[w\left(g_{i_{1}}, \ldots, g_{i_{n}}\right), g_{i_{n+1}}\right]^{-1} .
$$

Put

$$
J=\left\langle x, g_{i_{j}} \mid 1 \leq i \leq r, 1 \leq j \leq n+1\right\rangle .
$$

By [2, Lemma 2.7 (i)], the set $(J / Z(J))_{w}$ is finite. As $w$ is semiconcise, the subgroup $[w(J / Z(J)), J / Z(J)]$ is finite. Thus $v(J)$ has finite order modulo $Z(J)$, say $e$. Since $B \subseteq v(J)$, it follows that $b_{i}{ }^{e} \in Z(J)$ for all $i$. As $\langle x, B\rangle \leq J$, the result follows.

Proof of Theorem A. Set $v=\left[w\left(x_{1}, \ldots, x_{n}\right), x_{n+1}\right]$. Then $G$ is an $\mathrm{FC}(v)$-group by Lemma 2.3. Let $x$ be an arbitrary element of $G$. By Lemma 2.1, we can choose $b_{1}, \ldots, b_{r} \in G_{v}^{*}$ such that $x^{G_{v}^{*}}=\left\{x^{b_{1}}, \ldots, x^{b_{r}}\right\}$. Write $B=\left\{b_{1}, \ldots, b_{r}\right\}$. Define the order $<$ on the set of all (formal) products of the form $b_{i_{1}} \ldots b_{i_{j}}$, with $1 \leq i_{k} \leq r$ and $j \geq 1$, as follows. Put

$$
b_{i_{1}} \ldots b_{i_{j}}<b_{i_{1}^{\prime}} \ldots b_{i_{j^{\prime}}^{\prime}}
$$

if and only if one of the following conditions is satisfied: $j<j^{\prime}$, or $j=j^{\prime}$ and there is a positive integer $l \leq j$ such that $i_{l}<i_{l}^{\prime}$ and $i_{k}=i_{k}^{\prime}$ for all $k>l$.

Let $y$ be an arbitrary element of $v(G)$. Then $y=y_{1} \ldots y_{j}$, where each $y_{i} \in G_{v}^{*}$. By Lemma 2.2, for all $k \in\{1, \ldots, j\}$, there exists an integer $i_{k} \in\{1, \ldots, r\}$ such that $x^{y}=x^{b_{i_{1}} \ldots b_{i_{j}}}$. Clearly, we can choose $b_{i_{1}} \ldots b_{i_{j}}$ to be the smallest (in the sense of the order $<$ ) product of elements from $B$ such that $x^{y}=x^{b_{i_{1}} \ldots b_{i_{j}}}$. Let us now show that $i_{1} \geq i_{2} \geq \cdots \geq i_{j}$. Suppose to the contrary that $i_{k}<i_{k+1}$ for some $k$. Then

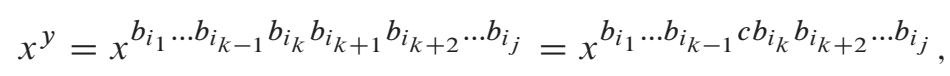

where $c=b_{i_{k}} b_{i_{k+1}} b_{i_{k}}^{-1} \in G_{v}^{*}$. In view of Lemma 2.2, we have

$$
x^{b_{i_{1}} \ldots b_{i_{k-1}} c}=x^{b_{i_{1}^{\prime}} \ldots b_{i_{k-1}^{\prime}} b_{i_{k+1}^{\prime}}}
$$

for some $1 \leq i_{1}^{\prime}, \ldots, i_{k-1}^{\prime}, i_{k+1}^{\prime} \leq r$ so that

$$
x^{y}=x^{b_{i_{1}^{\prime}} \ldots b_{i_{k-1}^{\prime}} b_{i_{k+1}^{\prime}} b_{i_{k}} b_{i_{k+2}} \ldots b_{i_{j}}} .
$$

This contradicts the choice of the product $b_{i_{1}} \ldots b_{i_{j}}$ because

$$
b_{i_{1}} \ldots b_{i_{k-1}} b_{i_{k}} b_{i_{k+1}} b_{i_{k+2}} \ldots b_{i_{j}}>b_{i_{1}^{\prime}} \ldots b_{i_{k-1}^{\prime}} b_{i_{k+1}^{\prime}} b_{i_{k}} b_{i_{k+2}} \ldots b_{i_{j}} .
$$


Thus $x^{y}=x^{b_{i_{1}} \ldots b_{i_{j}}}$ with $i_{1} \geq i_{2} \geq \ldots \geq i_{j}$ or, equivalently,

$$
x^{y}=x^{b_{r}{ }^{e r}} \ldots b_{1}{ }^{e_{1}}
$$

for some non-negative integers $e_{r}, \ldots, e_{1}$.

By Lemma 2.4, there exists a positive integer $e$ such that $b_{i}{ }^{e} \in Z(\langle x, B\rangle)$ for all $i$. Hence we may assume that $e_{i}<e$ for all $i$, and so $\left|x^{v(G)}\right| \leq e^{r}$. Thus $x^{v(G)}$ is finite for all $x \in G$. We conclude therefore that $v(G)=[w(G), G]$ is FC-embedded in $G$, as required.

\section{Proof of Theorem B}

The proof of Theorem B is very similar to that of Theorem A; the only important difference is the presence of bounds. In what follows, the term " $\{a, b, c, \ldots\}$ bounded" means "bounded from above by some function depending only on the parameters $a, b, c, \ldots$.

Before proving Theorem B, we recall briefly the ultraproduct construction of groups (see, for instance, [4] for more details).

For a non-empty set $I$, a filter over $I$ is a set $\mathcal{F}$ with the following properties:

(i) $\varnothing \notin \mathcal{F}, I \in \mathcal{F}$;

(ii) if $X, Y \in \mathcal{F}$, then $X \cap Y \in \mathcal{F}$;

(iii) if $X \in \mathscr{F}$ and $X \subseteq Y \subseteq I$, then $Y \in \mathscr{F}$.

The filter $\mathscr{F}$ is principal if there exists a non-empty set $Y \subseteq I$ such that

$$
\mathcal{F}=\{X \subseteq I \mid Y \subseteq X\},
$$

and non-principal otherwise. An example of a non-principal filter over an (infinite) set $I$ is the so-called cofinite filter

$$
\mathcal{F}=\{X \subseteq I \mid I \backslash X \text { is finite }\} .
$$

A filter $\mathcal{U}$ over $I$ is called an ultrafilter if, for every $X \subseteq I$, either $X \in \mathcal{U}$ or $I \backslash X \in U$. This is equivalent to saying that $U$ is a maximal filter over $I$. Also, $\mathcal{U}$ is a non-principal ultrafilter if and only if it contains the cofinite filter (see [4, Proposition 1.4]).

Given an ultrafilter $U$ over $I$ and a family $\left\{G_{i}\right\}_{i \in I}$ of groups, the ultraproduct modulo $\mathcal{U}$ is the quotient set of the Cartesian product $\prod_{i \in I} G_{i}$ with respect to the equivalence relation defined as follows: the tuples $\left(g_{i}\right)_{i \in I}$ and $\left(h_{i}\right)_{i \in I}$ of the Cartesian product are equivalent modulo $U$ if and only if

$$
\left\{i \in I \mid g_{i}=h_{i}\right\} \in \mathcal{U} .
$$


Thus the ultraproduct modulo $U$ can be seen as the quotient of the unrestricted direct product of groups $G_{i}$ by the subgroup consisting of all tuples $\left(g_{i}\right)_{i \in I}$ such that

$$
\left\{i \in I \mid g_{i}=1\right\} \in \mathcal{U} .
$$

The following is a consequence of Łoś's theorem (see [4, Corollary 3.2]), in the case of a sentence in the first-order language of groups.

Lemma 3.1. Let $\left\{G_{i}\right\}_{i \in I}$ be a family of groups, and let $U$ be an ultrafilter over I. Then a sentence in the first-order language of groups holds in the ultraproduct modulo $U$ if and only if the set of all $i \in I$ for which the sentence holds in $G_{i}$ is a member of $\mathcal{U}$.

Recall that the width of a group-word $w$ in a group $G$ is the supremum, as $g$ ranges over the verbal subgroup $w(G)$, of the minimum length of all decompositions of $g$ as a product of elements of $G_{w}^{*}$. Clearly, for a given positive integer $k$, the word $w$ has finite width at most $k$ in $G$ if and only if the product of any $k+1$ elements of $G_{w}^{*}$ can be expressed as a product of at most $k$ elements of $G_{w}^{*}$. Our next result relies on Lemma 3.1 and the following two facts, which have been stated in [5, proof of Theorem A.1]:

(a) for a given integer $m$, the property that a given word takes at most $m$ values in a group can be expressed as a sentence in the first-order language of groups;

(b) for a given positive integer $k$, the property that a given word has finite width at most $k$ in a group can be expressed as a sentence in the first-order language of groups.

Proposition 3.2. Let $m \geq 1$. Suppose that $w$ is a semiconcise word and $G$ is a group in which $w$ takes precisely $m$ values. Then the order of $[w(G), G]$ is m-bounded.

Proof. Assuming that $w$ involves $n$ variables, write $w=w\left(x_{1}, \ldots, x_{n}\right)$, and set $v\left(x_{1}, \ldots, x_{n}, x_{n+1}\right)=\left[w\left(x_{1}, \ldots, x_{n}\right), x_{n+1}\right]$. Then $[w(G), G]=v(G)$.

By way of contradiction, suppose there exists a family of groups $\mathscr{E}=\left\{G_{i}\right\}_{i \in \mathbb{N}}$ with the property that $\left|\left(G_{i}\right)_{w}\right| \leq m$ for all $i \in \mathbb{N}$ but

$$
\lim _{i \rightarrow \infty}\left|v\left(G_{i}\right)\right|=\lim _{i \rightarrow \infty}\left|\left[w\left(G_{i}\right), G_{i}\right]\right|=\infty .
$$

Consider a non-principal ultrafilter $\mathcal{U}$ over $\mathbb{N}$, and let $Q$ be the ultraproduct modulo $\mathcal{U}$ of $\mathcal{G}$. Then, by (a) and Lemma 3.1, we have $\left|Q_{w}\right| \leq m$. As $w$ is semiconcise, it follows that $v(Q)=[w(Q), Q]$ is finite. In particular, $v$ has finite width, say $k$, in $Q$. Now (b) and Lemma 3.1 yield that there exists $X \in \mathcal{U}$ such that $v$ has finite 
width at most $k$ in $G_{i}$ for all $i \in X$. Hence every element of $v\left(G_{i}\right)=\left[w\left(G_{i}\right), G_{i}\right]$ can be written as a product of at most $k$ elements of $\left(G_{i}\right)_{v}^{*}$. Moreover, as

$$
v\left(x_{1}, \ldots, x_{n}, x_{n+1}\right)=w\left(x_{1}, \ldots, x_{n}\right)^{-1} w\left(x_{1}, \ldots, x_{n}\right)^{x_{n+1}},
$$

from $\left|\left(G_{i}\right)_{w}\right| \leq m$, we get $\left|\left(G_{i}\right)_{v}\right| \leq m^{2}$ for all $i \in \mathbb{N}$. Therefore, $\left|v\left(G_{i}\right)\right| \leq m^{2 k}$ for all $i \in X$. As noted above, $\mathcal{U}$ contains the cofinite filter over $\mathbb{N}$, so $X \cap Y \in \mathcal{U}$ for every cofinite subset $Y$ of $\mathbb{N}$. In particular, $X \cap Y$ is non-empty. Therefore, every cofinite subset of $\mathbb{N}$ contains some element $i$ for which $\left|v\left(G_{i}\right)\right| \leq m^{2 k}$. This is incompatible with the assumption that $\left|v\left(G_{i}\right)\right|$ goes to infinity.

Lemma 3.3. Let $w=w\left(x_{1}, \ldots, x_{n}\right)$ be a group-word. If $G$ is a $\mathrm{BFC}(w)$-group such that $\left|x^{G_{w}}\right| \leq m$ for all $x \in G$, then the conjugacy class $x^{G_{w}^{*}}$ has $\{m, n\}$ bounded order for all $x \in G$.

Proof. By the second statement in [2, Proposition 2.9], a group $G$ is a $\operatorname{BFC}(w)$ group if and only if it is a $\operatorname{BFC}\left(w^{-1}\right)$-group. More precisely, if $G$ is a $\operatorname{BFC}(w)$ group such that $\left|x^{G_{w}}\right| \leq m$ for all $x \in G$, then $x^{G_{w^{-1}}}$ has $\{m, n\}$-bounded order. Since $G_{w}^{*}=G_{w} \cup G_{w^{-1}}$, the result follows.

Lemma 3.4. Let $w=w\left(x_{1}, \ldots, x_{n}\right)$ be a group-word, and set

$$
v=\left[w\left(x_{1}, \ldots, x_{n}\right), x_{n+1}\right] .
$$

If $G$ is a $\operatorname{BFC}(w)$-group such that $\left|x^{G} w\right| \leq m$ for all $x \in G$, then $G$ is a $\operatorname{BFC}(v)$ group and $x^{G_{v}}$ has $\{m, n\}$-bounded order for all $x \in G$.

Proof. This is similar to the proof of Lemma 2.3 taking into account that $x^{y}$ can only take $\{m, n\}$-boundedly many values by Lemma 3.3.

Lemma 3.5. Let $w=w\left(x_{1}, \ldots, x_{n}\right)$ be a group-word, and set

$$
v=\left[w\left(x_{1}, \ldots, x_{n}\right), x_{n+1}\right] .
$$

Let $G$ be a $\mathrm{BFC}(w)$-group such that $\left|x^{G_{w}}\right| \leq m$ for all $x \in G$, and let $B$ be a finite subset of $G_{v}^{*}$. Then, for any $x \in G$, there exists an $\{m, n,|B|\}$-bounded positive integer e such that $b^{e} \in Z(\langle x, B\rangle)$ for all $b \in B$.

Proof. It follows as in the proof of Lemma 2.4: by [2, Lemma 2.7 (ii)], the set $(J / Z(J))_{w}$ is finite of $\{m, n,|B|\}$-bounded order and, by Proposition 3.2, the number $e$ is $\{m, n,|B|\}$-bounded. 
Proof of Theorem B. Set $v=\left[w\left(x_{1}, \ldots, x_{n}\right), x_{n+1}\right]$. Then Lemma 3.4 tells us that $G$ is a $\operatorname{BFC}(v)$-group and $x^{G_{v}}$ has $\{m, n\}$-bounded order for all $x \in G$. Let $x$ be an arbitrary element of $G$, and choose

$$
b_{1}, \ldots, b_{r} \in G_{v}^{*} \quad \text { such that } x^{G_{v}^{*}}=\left\{x^{b_{1}}, \ldots, x^{b_{r}}\right\} .
$$

Write $B=\left\{b_{1}, \ldots, b_{r}\right\}$. Define the order $<$ on the set of all (formal) products of the form $b_{i_{1}} \ldots b_{i_{j}}$, with $1 \leq i_{k} \leq r$ and $j \geq 1$, as in (2.1) in the proof of Theorem A.

Let $y$ be an arbitrary element of $v(G)$. Arguing as in the proof of Theorem A, write

$$
x^{y}=x^{b_{r}{ }^{e_{r}} \ldots b_{1}{ }^{e_{1}}}
$$

for some non-negative integers $e_{r}, \ldots, e_{1}$. Since the number $r$ is $\{m, n\}$-bounded by Lemma 3.4, it follows from Lemma 3.5 that there exists an $\{m, n\}$-bounded positive integer $e$ such that $b_{i}{ }^{e} \in Z(\langle x, B\rangle)$ for all $i$. Hence we may assume that $e_{i}<e$ for all $i$, and so $\left|x^{v(G)}\right| \leq e^{r}$. Thus $x^{v(G)}$ is finite of $\{m, n\}$-bounded order for all $x \in G$, and so $v(G)$ is BFC-embedded in $G$.

\section{Examples}

In this section, we first provide some new examples of semiconcise words, and then we give an example of a word which is not semiconcise.

The following lemma is well known (see, for instance, [10, Lemma 4.28]).

Lemma 4.1. Let $G=\left\langle g_{1}, \ldots, g_{m}\right\rangle$ be a group, and suppose that, for a groupword $w$, the set $\left\{\left[x, g_{i}\right] \mid x \in G_{w}, i=1, \ldots, m\right\}$ is finite. Then $G_{w}$ is contained in finitely many right cosets of $w(G) \cap Z(G)$.

Proof. Suppose that there exists an infinite sequence $x_{1}, x_{2}, \ldots$ of elements of $G_{w}$ belonging to distinct right cosets of $C=w(G) \cap Z(G)$. For any $i=1, \ldots, m$, put $S_{i}=\left\{\left[x, g_{i}\right] \mid x \in G_{w}\right\}$. Denote by $P$ the Cartesian product $S_{1} \times \cdots \times S_{m}$, and let $\pi$ be the map sending $x \in G_{w}$ to $\left(\left[x, g_{1}\right], \ldots,\left[x, g_{m}\right]\right) \in P$. Now we have that $x_{i} \pi=x_{j} \pi$ implies $x_{i} x_{j}^{-1} \in C$, or $C x_{i}=C x_{j}$ which is impossible. It follows that $P$ must be infinite, a contradiction.

Proposition 4.2. Let $w=w\left(x_{1}, \ldots, x_{n}\right)$ be a group-word, and set

$$
v=\left[w\left(x_{1}, \ldots, x_{n}\right), x_{n+1}\right] \text {. }
$$

If $w$ is semiconcise, then $v$ is semiconcise. 
Proof. Let $G$ be a group, and assume that $G_{v}$ is finite. Since $v(G)^{\prime}$ is finite (see, for instance, [6, Proposition 1]), we may assume that $v(G)$ is abelian. It follows that every subgroup of $v(G)$ is finitely generated. Let $K=\left\langle g_{1}, \ldots, g_{m}\right\rangle$ be a finitely generated subgroup of $G$ such that $v(G)=v(K)$. For an arbitrary $g=g_{0} \in G$, put $H=\left\langle g_{0}, K\right\rangle$. Of course, $v(G)=v(H)$. Also, $\left|H: C_{H}(v(H))\right|$ is finite because every $h \in H_{v}$ has only finitely many conjugates in $H$. We claim that $|v(H): v(H) \cap Z(H)|$ is also finite, from which it follows that $[v(H), H]$ is finite by a result of Baer (see [10, Corollary, p. 103]). In fact, the set

$$
\left\{\left[x, g_{i}\right] \mid x \in H_{w}, i=0,1, \ldots, m\right\}
$$

is finite, and therefore, by Lemma $4.1, H_{w}$ is contained in finitely many right cosets of $w(H) \cap Z(H)$. Hence $(H / Z(H))_{w}$ is finite. Since $w$ is semiconcise, we obtain that

$$
[w(H), H] Z(H) / Z(H) \simeq v(H) / v(H) \cap Z(H)
$$

is finite.

Then $[v(H), H]=[v(G), H]$ is finite. In particular, $[v(G),\langle g\rangle]$ is finite for any $g \in G$. Thus $[v(G), G]$ is a finitely generated periodic abelian group, and so $[v(G), G]$ is finite. This proves that $v$ is semiconcise.

As an immediate consequence of Proposition 4.2, we get new examples of semiconcise words starting from Engel words, which are semiconcise by [6, Proposition 4].

Corollary 4.3. Let $w=[x, n y]$ be the $n$-Engel word, and set

$$
v=\left[x, n y, z_{1}, \ldots, z_{m}\right],
$$

where the variables $x, y, z_{1}, \ldots, z_{m}$ are all different. Then $v$ is semiconcise.

According to [8], for any odd integer $n>10^{10}$ and any prime number $p>5000$, the word

$$
v(x, y)=\left[\left[x^{p n}, y^{p n}\right]^{n}, y^{p n}\right]^{n}
$$

is not concise. Indeed, Ivanov constructed a 2-generator torsion-free group $A$, whose center is cyclic and $A / Z(A)$ is infinite of exponent $p^{2} n$, such that $v$ takes only two values in $A$ and the nontrivial value is a generator of $Z(A)$.

In [1, Section 4], the authors considered a modification of Ivanov's example, namely the wreath product

$$
G=A \mathrm{wr} B
$$


where $A$ is as above and $B=\langle b\rangle$ is a cyclic group of order 2. Furthermore, taking

$$
w(x, y)=v\left(x^{2}, y^{2}\right),
$$

they showed that $\left|G_{w}\right| \leq 4$ and $b^{w(G)}$ is infinite. In a similar way, we now prove that $b^{[w(G), G]}$ is also infinite. This implies that $w$ is not semiconcise.

Proposition 4.4. There exist a group-word $w$ (which is not semiconcise) and a $\mathrm{BFC}(w)$-group $G$ such that $[w(G), G]$ is not $\mathrm{FC}$-embedded in $G$.

Proof. Let $G$ and $w$ be as in (4.1) and (4.2), respectively. Then, by [1, Proposition 4.1], $G$ is a $\operatorname{BFC}(w)$-group.

Denote by $K=A \times A^{b}$ the base group of $G$. For any odd integer $m \geq 1$, let $N=\left\langle v_{0}^{m},\left(v_{0}^{b}\right)^{m}\right\rangle$, where $v_{0} \in A$ is the nontrivial value of $v(x, y)$ in $A$. Notice that $N$ is central in $K$ and closed under conjugation by $b$ so that $N$ is a normal subgroup of $G$. Also, since $K / N$ has odd exponent $p^{2} m n$ and $|G / K|=2$, we have

$$
K / N=\left\{g^{2} N \mid g \in G\right\}
$$

and consequently

$$
(K / N)_{v}=(G / N)_{w} .
$$

Hence $v_{0} N \in(G / N)_{w}$, and therefore $v_{0}^{k} N \in w(G / N)$ for any integer $k$. It follows that

$$
b^{\left[b, v_{0}^{k}\right]} N \in(b N)^{[w(G / N), G / N]} .
$$

Now

$$
b^{\left[b, v_{0}^{k}\right]}=b\left[b, v_{0}^{k}, b\right]^{-1}=b\left[\left(v_{0}^{b}\right)^{-k} v_{0}^{k}, b\right]^{-1}=b\left(v_{0}^{2}\left(v_{0}^{b}\right)^{-2}\right)^{k},
$$

where $v_{0}^{2}\left(v_{0}^{b}\right)^{-2}$ has order $m$ in $G / N$. Thus

$$
\left|\left\{b^{\left[b, v_{0}^{k}\right]} N \mid k \in \mathbb{Z}\right\}\right|=m,
$$

and so

$$
\left|(b N)^{[w(G / N), G / N]}\right| \geq m .
$$

In particular, $\left|b^{[w(G), G]}\right| \geq m$. Since $m$ is an arbitrary odd positive integer, we conclude that $b^{[w(G), G)]}$ is infinite. This proves that $\left.[w(G), G)\right]$ is not $\mathrm{FC}(w)$ embedded in $G$.

Acknowledgments. The authors would like to thank the referee for a number of useful suggestions and, in particular, for correcting a mistake in an earlier version of the proof of Proposition 3.2. 


\section{Bibliography}

[1] S. Brazil, A. Krasilnikov and P. Shumyatsky, Groups with bounded verbal conjugacy classes, J. Group Theory 9 (2006), no. 1, 127-137.

[2] C. Delizia, P. Shumyatsky and A. Tortora, On groups with finite conjugacy classes in a verbal subgroup, Bull. Aust. Math. Soc. 96 (2017), no. 3, 429-437.

[3] C. Delizia, P. Shumyatsky, A. Tortora and M. Tota, On conciseness of some commutator words, Arch. Math. (Basel) 112 (2019), no. 1, 27-32.

[4] P. C. Eklof, Ultraproducts for algebraists, in: Handbook of Mathematical Logic, Stud. Logic Found. Math. 90, North-Holland, Amsterdam (1977), 105-137.

[5] G. A. Fernández-Alcober and M. Morigi, Outer commutator words are uniformly concise, J. Lond. Math. Soc. (2) 82 (2010), no. 3, 581-595.

[6] G. A. Fernández-Alcober, M. Morigi and G. Traustason, A note on conciseness of Engel words, Comm. Algebra 40 (2012), no. 7, 2570-2576.

[7] S. Franciosi, F. de Giovanni and P. Shumyatsky, On groups with finite verbal conjugacy classes, Houston J. Math. 28 (2002), no. 4, 683-689.

[8] S. V. Ivanov, P. Hall's conjecture on the finiteness of verbal subgroups, Izv. Vyssh. Uchebn. Zaved. Mat. (1989), no. 6, 60-70.

[9] A. Y. Olshanskiŭ, Geometry of Defining Relations in Groups, Kluwer Academic, Dordrecht, 1991.

[10] D. J.S. Robinson, Finiteness Conditions and Generalized Soluble Groups. Part 2, Springer, New York, 1972.

[11] J. Wilson, On outer-commutator words, Canadian J. Math. 26 (1974), 608-620.

Received July 24, 2019; revised February 7, 2020.

\section{Author information}

Costantino Delizia, Dipartimento di Matematica, Università di Salerno,

Fisciano (SA), 84084, Italy.

E-mail: cdelizia@unisa.it

Pavel Shumyatsky, Department of Mathematics, University of Brasilia,

Brasilia-DF, 70910-900, Brazil.

E-mail:pavel@unb.br

Antonio Tortora, Dipartimento di Matematica e Fisica,

Università della Campania “Luigi Vanvitelli", Caserta, 81100, Italy.

E-mail: antonio.tortora@unicampania.it 